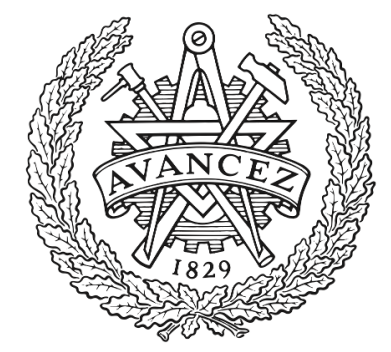

\title{
CHALMERS
}

UNIVERSITY OF TECHNOLOGY

\section{Variable Structure Control of Engine Idle Speed with Estimation of Unmeasurable Disturbances}

Downloaded from: https://research.chalmers.se, 2023-04-26 10:09 UTC

Citation for the original published paper (version of record):

Stotsky, A., Egardt, B., Eriksson, S. (2000). Variable Structure Control of Engine Idle Speed with Estimation of Unmeasurable Disturbances. Journal of Dynamic Systems, Measurement, and Control, 122(4): 599-603

N.B. When citing this work, cite the original published paper. 


\section{A. Stotsky \\ Volvo Car Corporation, \\ Dept. 96566 HC2N, \\ SE-405 08 Gothenburg, Sweden e-mail: vcc9.stotsky@memo.volvo.se \\ B. Egardt \\ Department of Signals and Systems, \\ Variable Structure Control of Engine Idle Speed With Estimation of Unmeasurable Disturbances} Control Engineering Laboratory, Chalmers University of Technology, SE-412 96 Gothenburg, Sweden e-mail: be@s2.chalmers.se

\section{S. Eriksson}

Volvo Car Corporation,

Dept. 96560 HB2S,

SE-405 08 Gothenburg, Sweden

e-mail: vcet.96560se@memo.volvo.se

\begin{abstract}
A new controller for throttle and spark advance to control the engine speed at idle under unknown time varying disturbances is proposed in this paper. By using measurements of the engine speed the disturbance estimator is designed to reconstruct a disturbance torque. The controller is formulated so that the throttle is used as much as possible as a main tool to produce a torque and spark advance is used to compensate intake to torque production delay. The stability of the system is proved via Lyapunov function method. [S0022-0434(00)01304-6]
\end{abstract}

Keywords: Automotive Control, Idle Speed Control, Engine Model, Variable Structure Control, Time Delay Systems

\section{Introduction}

The main source of the performance deterioration of the idle speed control (ISC) systems is disturbances such as rapid external load changes and slow varying changes in operating conditions. External load changes are the result of loading due to the air conditioning, battery charging etc. Very often such disturbances are measurable and feedforward compensation is used to improve the performance. However, the loads applied to the engine may change in time and more power consuming equipment may be installed in a vehicle after it leaves the factory. In this case one must consider the ISC problem under unmeasurable disturbances.

Although ISC is a well-studied topic, see for example the comprehensive survey [1] and references therein, many works ignore intake to torque production delay, which is essential in this case [2] or based on linearized engine model, which is valid only locally.

This paper presents a new approach to the ISC problem based on a second-order nonlinear engine model, which takes into account intake to torque production delay and unmeasurable time varying disturbances.

Two inputs may be used to control the engine speed at idle: throttle and spark advance. Throttle provides large variations of torque without increasing exhaust emissions; however, the main problem of using throttle as a control is intake to torque production delay. Spark advance can be seen as a fast control; however, it produces limited torque and increases both emissions and fuel consumption. In this paper, we propose an innovative solution for ISC, which utilizes advantages and compensates disadvantages of both controls. A similar combined spark/throttle control law was proposed in [3] (see also [4]). However, the stability proof is based on the assumption that the spark influence is constant when deriving the throttle control law. In other words, two controls are treated independently and it is not practical in many cases.

The key idea of our control law is the following. First, we design a high gain disturbance estimator, for estimation of unknown disturbances, such that the upper bound of the estimation error can be made arbitrarily small (this error is compensated by spark advance). A control law is designed such that the throttle is used as a main tool to produce the torque, while spark is used both

Contributed by the Dynamic Systems and Control Division for publication in the JouRnal OF DYNAMIC Systems, MEASUREMENT, AND CONTROL. Manuscript received by the Dynamic Systems and Control Division February 9, 2000. Associate Technical Editors: E. Misawa and V. Utkin. for compensation of throttle to torque delay and for variable structure feedback that compensates the disturbance estimation error. Since the retarded spark increases the exhaust emissions, our control law drives spark advance to MBT (minimum spark advance for the best torque), if the engine speed is close to the desired engine speed and there is no need for fast control.

It is worth remarking that similar disturbance estimation technique was proposed in [5,6] for discrete time case and in [7] for continuous time adaptive control.

The paper is organized as follows. In the next section the engine model is described. Problem statement is given in Section 3. In Section 4 we design our control law, i.e., observer for the disturbance torque, and control laws for spark advance and throttle. Section 5 is devoted to the stability analysis of the whole system. Simulation results are presented in Section 6 and we finish with brief concluding remarks in Section 7.

\section{Engine Model}

In this section we develop the engine model which is based on the results presented in [8,9] and [10].

The first equation is obtained by considering the conservation of mass

$$
\dot{m}_{a}=\dot{m}_{\mathrm{maf}}-\dot{m}_{\mathrm{cyl}}
$$

where $m_{a}$ is the mass of air in the intake manifold, $\dot{m}_{\text {maf }}$ is the mass rate of air entering the manifold, and $\dot{m}_{\text {cyl }}$ is the mass rate of air leaving the manifold and entering the combustion chamber. The mass rate of air entering the manifold is modeled as

$$
\dot{m}_{\text {maf }}=a_{1} u_{1} p_{r}
$$

where $a_{1}=0.3861 \mathrm{~kg} / \mathrm{s}$ for the engine of interest, $u_{1}$ is normalized throttle characteristic [0:1], $p_{r}$ is normalized pressure influence

$$
p_{r}=\left\{\begin{array}{l}
\sqrt{\left(\frac{p}{p_{0}}\right)^{1.428}-\left(\frac{p}{p_{0}}\right)^{1.714}} \\
0.259 \text { otherwise }
\end{array} \text { if } \frac{p}{p_{0}}>0.528\right.
$$

where $p$ is the pressure in the intake manifold, $p_{0}$ is atmospheric pressure $\left(p_{0}=1 \mathrm{Bar}\right)$.

Pressure $p$ in the intake manifold changes according to the following equation, which comes from the differentiation of the ideal gas law under the assumption that the intake manifold temperature is constant, 


$$
\dot{p}=k_{m}\left(\dot{m}_{\mathrm{maf}}-\dot{m}_{\mathrm{cyl}}\right)
$$

where

$$
k_{m}=\frac{R T}{V_{m}}
$$

where $R=287.9 \mathrm{Nm} / \mathrm{Kg} / \mathrm{K}$ is a gas constant, $T$ is the intake manifold temperature, $T=318^{\circ} \mathrm{K}\left(45^{\circ} \mathrm{C}\right), V_{m}=5 \cdot 10^{-3} \mathrm{~m}^{3}$ is intake manifold volume.

We found it convenient to use the pressure ratio $\left(p / p_{0}\right)$ as a state variable. The pressure ratio $\left(p / p_{0}\right)$ in the intake manifold changes according to the following equation

$$
\frac{\dot{p}}{p_{0}}=k_{1}\left(\dot{m}_{\mathrm{maf}}-\dot{m}_{\mathrm{cyl}}\right)
$$

where we denoted

$$
k_{1}=\frac{T}{V_{m} T_{0} \rho_{0}}
$$

where $\rho_{0}$ is the atmospheric density and $T_{0}$ is the corresponding atmospheric temperature. In our case $\rho_{0}=1.2 \mathrm{~kg} / \mathrm{m}^{3}$ and $T_{0}$ $=288^{\circ} \mathrm{K}\left(15^{\circ} \mathrm{C}\right)$.

The mass flow rate entering the combustion chamber satisfies the following equation which comes from the speed density calculation $[8,10]$

$$
\dot{m}_{\mathrm{cyl}}=k \omega \frac{p}{p_{0}}
$$

where we denoted

$$
k=\frac{\rho_{0} T_{0} V_{\mathrm{cyl}} \eta}{4 \pi T}
$$

where $\omega$ is the engine speed $(\mathrm{rad} / \mathrm{s}), \eta$ is a volumetric efficiency (for simplicity we took the constant value of efficiency, $\eta=0.8$ ), $V_{\text {cyl }}=2.5 \cdot 10^{-3} \mathrm{~m}^{3}$ is the volume of five cylinders of the engine, $\pi=3.1416$.

The rotational dynamics of the engine is modeled as

$$
J_{e} \dot{\omega}=T_{\mathrm{ind}}-T_{f}-T_{d}-T_{p}
$$

where $T_{\text {ind }}$ is indicated torque, $T_{f}$ is friction torque, $T_{p}$ is pump torque, and $T_{d}$ is disturbance torque, $J_{e}=0.255 \mathrm{kgm}^{2}$ is the inertia moment of the engine.

Indicated Engine Torque is the following

$$
T_{\text {ind }}=a_{2} \frac{\dot{m}_{\mathrm{cyl}}\left(t-t_{d}\right)}{\omega\left(t-t_{d}\right)} a_{f i}\left(t-t_{d}\right) f_{a}\left(t-t_{s}\right)
$$

where $a_{2}=8.51 \cdot 10^{5} \mathrm{Nm} / \mathrm{kg} / \mathrm{rad}$ represents the maximum torque capacity, $a_{f i}\left(t-t_{d}\right)$ is normalized air to fuel influence (in this paper we assume that air to fuel ratio is under control and $a_{f i}(t$ $\left.\left.-t_{d}\right)=1\right), f_{s}\left(t-t_{s}\right)$ is a function of spark influence.

Substituting (7) in (10) we get the following expression for indicated torque

$$
T_{\text {ind }}=a_{2} k \frac{p\left(t-t_{d}\right)}{p_{0}} f_{s}\left(t-t_{s}\right)
$$

At low engine speeds MBT (minimum spark advance for the best torque) is 20 degrees before top dead center and it is possible to retard spark up to 10 degrees after top dead center. This means that the total range for spark advance/retard is 30 degrees or \pm 15 if spark is retarded by 15 degrees from MBT.

The following curve may be used [5] for the spark influence

$$
f_{s}=\left(\cos \left(-b+u_{2}\right)\right)^{2.875}
$$

where $b$ is the position of OY axis from MBT (see Fig. 1), measured in radians, and $u_{2}$ is our control. Notice, that the distance $b$

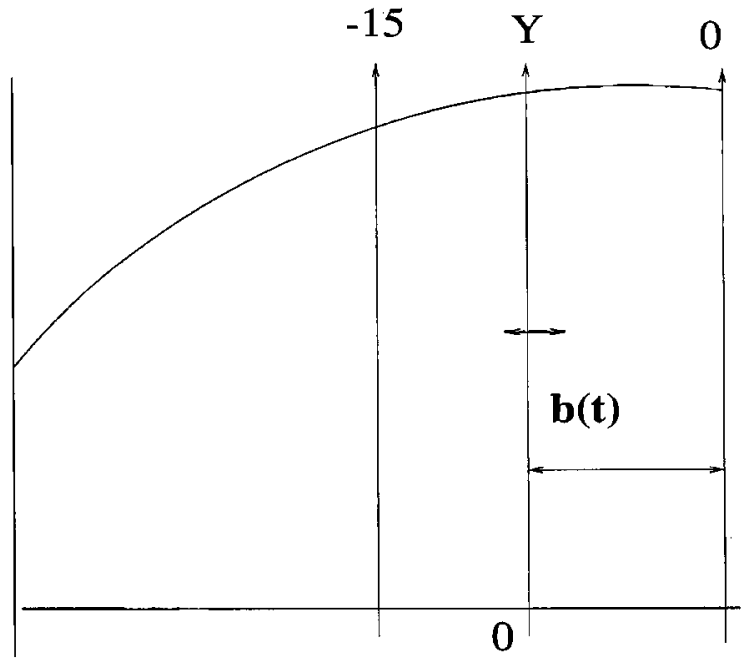

Spark Advance from MBT

MBT

Fig. 1

can be fixed or adjusted within the interval $\left[0,15^{\circ}\right]$, thereby control action $u_{2}$ varies within the interval $[-b, b]$. If $b$ is fixed and $u_{2}=0$ then spark is still retarded from MBT. Unfortunately, retarding spark increases emissions, coefficient of variation of engine torque, and hence partial burns and misfires. Moreover, retarding spark negatively affects the fuel economy. On the other hand, when the engine speed is close enough to the desired engine speed there is no need for spark as a control and it should be kept at MBT whenever possible. By adjusting the distance $b$ it is possible to drive the spark advance to MBT when engine speed is close to the desired engine speed. On the other hand, if the engine speed mismatch is big enough $b$ should be kept at $15^{\circ}$ to provide the largest range for control.

Since the engine torque production is a discrete process, but modeled in the continuous time domain we must introduce the following delays $[8]$ :

$$
\begin{aligned}
t_{d} & =\frac{5.48}{\omega} \\
t_{s} & =\frac{1.3}{\omega}
\end{aligned}
$$

where $t_{d}$ is intake to torque production delay, and $t_{s}$ is spark to torque production delay. As can be seen from (13) and (14) intake to torque production delay is more than four times larger than spark to torque delay. As we observed by simulations, spark to torque delay can be neglected, see also [11].

Engine Friction Torque can be modeled as

$$
T_{f}=\left(a_{1 f}+a_{2 f} \omega+a_{3 f} \omega^{2}\right) \frac{V_{1 \mathrm{cyl} 1} 1000 z}{4 \pi}
$$

where $a_{1 f}=97 \mathrm{~N} / \mathrm{m}^{2}, a_{2 f}=0.1432 \mathrm{~N} / \mathrm{m}^{2}(\mathrm{~s} / \mathrm{rad}), a_{3 f}=2.74 \cdot 10^{-4}$ $\mathrm{N} / \mathrm{m}^{2}\left(\mathrm{~s}^{2} / \mathrm{rad}^{2}\right), V_{1 \mathrm{cyl}}=0.5 \cdot 10^{-3} \mathrm{~m}^{3}$ is the volume of one cylinder, $z=5$ is the number of cylinders.

Engine Pump Torque can be modeled as

$$
T_{p}=\left(p_{0}-p\right) \frac{V_{1 \mathrm{cyl} z}}{4 \pi}
$$

Engine Disturbance Torque $T_{d}$ can be presented, for idle control problem, by a class of bounded functions with bounded derivatives, i.e.,

$$
\left|T_{d}(t)\right| \leqslant c, \quad\left|\dot{T}_{d}(t)\right| \leqslant c_{1}
$$

where $c$ and $c_{1}$ are positive constants. Typical value for $c$ is 20 $\mathrm{Nm}$. 
Finally, the engine model can be written as follows:

$$
\begin{gathered}
J_{e} \dot{\omega}=a_{2} k \frac{p\left(t-t_{d}\right)}{p_{0}}\left(\cos \left(-b+u_{2}\right)\right)^{2.875}-T_{f}-T_{d}-T_{p} \\
\dot{m}_{\mathrm{cyl}}=k \omega \frac{p}{p_{0}} \\
\frac{\dot{p}}{p_{0}}=k_{1}\left(a_{1 s} u_{1}-\dot{m}_{\mathrm{cyl}}\right)
\end{gathered}
$$

where $T_{f}$ and $T_{p}$ are given by (15) and (16), $k$ and $k_{1}$ are given by (8) and (6), we denoted $a_{1 s}=a_{1} p_{r}$. Our next step is to present the problem statement.

\section{Problem Statement}

It is well known that the engine at idle is very sensitive to the disturbance torques, and very often disturbances are unmeasurable so that feedforward compensation cannot be applied.

Our problem is to find controls $u_{1}$ and $u_{2}$ (throttle and spark advance) so that, to achieve the following control aim:

$$
\lim _{t \rightarrow \infty}\left|\omega(t)-\omega_{d}\right|=0
$$

where $\omega_{d}=100 \mathrm{rad} / \mathrm{s}$ is the idle speed, under unknown time varying disturbances $T_{d}(t)$. We measure the engine speed $\omega$ and pressure $p$ in the intake manifold. Friction torque and pump torque can be computed as well using measurements of the engine speed and pressure, see (15) and (16). Modeling errors in pump and friction torques can be considered as a disturbance torque as well. Engine parameters are assumed to be known. The disturbance torque is unknown and unmeasurable, but it is assumed that it is bounded with bounded derivative.

\section{Control Design}

4.1 Observer Design for $T_{d}(t)$. Define the following estimation error

$$
e=\alpha_{0} J_{e} \omega-\varepsilon+T_{d}(t)
$$

where $\alpha_{0}>0$ and $\varepsilon(t)$ is the solution of the following differential equation

$$
\begin{aligned}
\dot{\varepsilon}= & -\alpha_{0} \varepsilon+\alpha_{0} a_{2} k \frac{p\left(t-t_{d}\right)}{p_{0}}\left(\cos \left(-b+u_{2}\right)\right)^{2.875} \\
& -\alpha_{0} T_{f}-\alpha_{0} T_{p}+\alpha_{0}^{2} J_{e} \omega
\end{aligned}
$$

Our next aim is to show that the upperbound of the error $|e(t)|$ can be made arbitrarily small. Choosing the following Lyapunov like function

$$
V=\frac{1}{2} e^{2}
$$

we evaluate its derivative along the solutions of the system (18), (22), (23):

$$
\dot{V}=-\alpha_{0} e^{2}+e \dot{T}_{d} \leqslant-\alpha_{0} V+\frac{c_{1}^{2}}{2 \alpha_{0}}
$$

and the following bound for the estimation error is true:

$$
|e(t)| \leqslant \sqrt{e^{2}(0) e^{-\alpha_{0} t}+\frac{c_{1}^{2}}{\alpha_{0}^{2}}}
$$

The bound (26) guarantees that the estimation error can be made arbitrarily small by amplifying the design parameter $\alpha_{0}$. Then from (22) we get the estimate of the disturbance torque

$$
T_{d}(t)=-\alpha_{0} J_{e} \omega+\varepsilon+e(t)
$$

where $e(t)$ can be made arbitrarily small. Notice that $e(t) \rightarrow 0$ if $T_{d}=$ const.

Notice, that the way of the estimation of unknown disturbances presented above is equivalent to the estimation of $\dot{\omega}$ via a "dirty differentiator." In this case $T_{d}$ can be computed via (18).

Substituting (27) in (18) we get the following:

$$
\begin{gathered}
J_{e} \dot{\omega}=a_{2} k \frac{p\left(t-t_{d}\right)}{p_{0}}\left(\cos \left(-b+u_{2}\right)\right)^{2.875} \\
-T_{f}-T_{p}+\alpha_{0} J_{e} \omega-\varepsilon-e(t) \\
\dot{m}_{\mathrm{cyl}}=k \omega \frac{p}{p_{0}} \\
\frac{\dot{p}}{p_{0}}=k_{1}\left(a_{1 s} u_{1}-\dot{m}_{\mathrm{cyl}}\right)
\end{gathered}
$$

4.2 Control Law Design for Spark Advance. Define the spark advance, $u_{2}$, as a solution of the following algebraic equation:

$$
\begin{aligned}
p\left(t-t_{d}\right)\left(\cos \left(-b+u_{2}\right)\right)^{2.875}= & p(t)\left(\cos \left(-b+u_{2 b}\right)\right)^{2.875} \\
& -\gamma_{s} \operatorname{sign}\left(\omega-\omega_{d}\right)
\end{aligned}
$$

where $u_{2 b}=0$ corresponds to the case where spark advance is not controlled, $\gamma_{s}>0$. The physical meaning of the spark advance control gets clear if we substitute (31) into (28). Then

$$
\begin{aligned}
J_{e} \dot{\omega}= & \frac{a_{2} k p(t) c_{a}}{p_{0}}-\frac{a_{2} k \gamma_{s}}{p_{0}} \operatorname{sign}\left(\omega-\omega_{d}\right)-T_{f}-T_{p} \\
& +\alpha_{0} J_{e} \omega-\varepsilon-e(t)
\end{aligned}
$$

where $\left.c_{a}=\cos \left(-b+u_{2 b}\right)\right)^{2.875}$. It is clear that the first term in the right-hand side of (31) compensates the time delay $t_{d}$ for $p(t$ $-t_{d}$ ), and the second term gives relevant variable structure feedback which drives $\omega$ to $\omega_{d}$. Unfortunately, the spark influence on the torque production is limited and $\gamma_{s}$ should be chosen sufficiently small.

Resolving (31) with respect to the control we get

$$
u_{2}=b-\arccos \left(\left\{\frac{p(t)}{p\left(t-t_{d}\right)} c_{a}-\frac{\gamma_{s}}{p\left(t-t_{d}\right)} \operatorname{sign}\left(\omega-\omega_{d}\right)\right\}^{1 / 2.875}\right)
$$

Notice that, if there is no time delay $t_{d}=0$, and $\omega=\omega_{d}$ then $u_{2}$ $=u_{2 b}$ and spark is retarded on $b$ radians from MBT.

Remark: As was already mentioned the distance $b$ from MBT to OY axis (see Fig. 1), can be fixed or adjusted. Below we propose the following adjustment law for the moving axis:

$$
\dot{b}=-\frac{1}{\tau}\left(b-0.2618 \operatorname{sat}\left(\omega-\omega_{d}\right)\right), \quad b(0)=0
$$

where $\tau>0, b$ is measured in radians and $\operatorname{sat}\left(\omega-\omega_{d}\right)$ is the following saturation function

$$
\operatorname{sat}\left(\omega-\omega_{d}\right)=\left\{\begin{array}{l}
\frac{1}{\lambda_{0}\left|\omega-\omega_{d}\right|} \text { if }\left|\omega-\omega_{d}\right|<\lambda_{0} \\
1 \quad \text { otherwise }
\end{array}\right.
$$

where $\lambda_{0}>0$ is the size of the boundary layer. Finally, we have to limit our spark advance taking into account the adjustable offset. Redenoting $u_{2}$ defined in (33) as $u_{20}$, the bounded spark control law is the following:

$$
u_{20}=b-\arccos \left(\left\{\frac{p(t)}{p\left(t-t_{d}\right)} c_{a}-\frac{\gamma_{s}}{p\left(t-t_{d}\right)} \operatorname{sign}\left(\omega-\omega_{d}\right)\right\}^{1 / 2.875}\right)
$$




$$
u_{2}=\left\{\begin{array}{l}
b \quad \text { if } u_{20} \geqslant b \\
u_{20} \text { if }-b \leqslant u_{20} \leqslant b \\
-b \quad \text { if } u_{20} \leqslant-b
\end{array}\right.
$$

The physical meaning of the adjustable axis is the following. If $\left(\omega-\omega_{d}\right)$ is out of the boundary layer $\lambda_{0}$, then the axis moves to the 15 degrees $(0.2618 \mathrm{rad})$ from MBT, exponentially, according to the differential equation (34) with the rate $1 / \tau$. The position of the axis at $15^{\circ}$ corresponds to the maximal control power. If ( $\omega$ $\left.-\omega_{d}\right)$ is within the boundary layer $\lambda_{0}$ then $b$ is adjusted as follows

$$
\dot{b}=-\frac{1}{\tau}\left(b-\frac{0.2618}{\lambda_{0}}\left|\omega-\omega_{d}\right|\right)
$$

and as soon as $\left(\omega(t)-\omega_{d}\right) \rightarrow 0$ then $b$ and $u_{2}$ tend to zero, and spark advance tends to MBT. Notice that, only the sum $(-b$ $+u_{2}$ ) defines the position of the spark advance from MBT (if $-b+u_{2}=0$ then the spark advance is at MBT).

Due to the choice of the spark advance the system (28), (30) can be written in the following form:

$$
\begin{gathered}
J_{e} \dot{\omega}=\frac{a_{2} k p(t) c_{a}}{p_{0}}-\frac{a_{2} k \gamma_{s}}{p_{0}} \operatorname{sign}\left(\omega-\omega_{d}\right)-T_{f} \\
-T_{p}+\alpha_{0} J_{e} \omega-\varepsilon-e(t) \\
\dot{m}_{\mathrm{cyl}}=k \omega \frac{p}{p_{0}} \\
\frac{\dot{p}}{p_{0}}=k_{1}\left(a_{1 s} u_{1}-\dot{m}_{\mathrm{cyl}}\right)
\end{gathered}
$$

4.3 Control Law Design for Throttle. First we define the desired pressure ratio $\left(p / p_{0}\right) d$,

$$
\left(\frac{p}{p_{0}}\right)_{d}=\frac{1}{c_{a} a_{2} k}\left\{-\alpha_{1} \widetilde{\omega}+T_{f}+T_{p}+\varepsilon-\alpha_{0} J_{e} \omega\right\}
$$

where $\widetilde{\omega}(t)=\omega-\omega_{d}, \alpha_{1}>0$. The physical meaning of the desired pressure ratio $\left(p / p_{0}\right)_{d}$ gets clear if we suppose at a time instant that $p / p_{0}=\left(p / p_{0}\right)_{d}$, then substituting (40) into (37), we get

$$
J_{e} \dot{\omega}=-\alpha_{1} \widetilde{\omega}-\frac{a_{2} k \gamma_{s}}{p_{0}} \operatorname{sign} \widetilde{\omega}-e(t)
$$

Notice, that the upper bound of the estimation error $e(t)$ can be made arbitrarily small, so that even small $\gamma_{s}$ is able to compensate the estimation error.

Now our aim is to choose the throttle position so as to drive the pressure ratio to the desired one. Substituting (38) in (39) we get the following equation for the pressure evolution:

$$
\frac{\dot{p}}{p_{0}}=k_{1} a_{1 s} u_{1}-k_{1} k \omega \frac{p}{p_{0}}
$$

Choosing the throttle position as follows

$$
u_{1}=\frac{1}{a_{1 s}} k \omega \frac{p}{p_{0}}+\frac{1}{a_{1 s} k_{1}}\left(-\alpha_{2}\left(\frac{p}{p_{0}}-\left(\frac{p}{p_{0}}\right)_{d}\right)+\left(\frac{\dot{p}}{p_{0}}\right)_{d}\right)
$$

we get

$$
\frac{\dot{p}}{p_{0}}-\left(\frac{\dot{p}}{p_{0}}\right)_{d}=-\alpha_{2}\left(\frac{p}{p_{0}}-\left(\frac{p}{p_{0}}\right)_{d}\right)
$$

where $\alpha_{2}>0$.

Notice, that the implementation of (43) requires measurements of $\left(\dot{p} / p_{0}\right)_{d}$. The derivative can be estimated via a sliding mode observer which guarantees the convergence of the estimation error in a finite time, see [12] and [13] for details.

\section{Stability Analysis of the System}

Finally, the error model of the system can be written in the following form:

$$
\begin{gathered}
J_{e} \dot{\tilde{\omega}}=-\alpha_{1} \widetilde{\omega}-\frac{a_{2} k \gamma_{s}}{p_{0}} \operatorname{sign} \widetilde{\omega}+c_{a} a_{2} e_{p}-e(t) \\
\dot{e}_{p}=-\alpha_{2} e_{p}
\end{gathered}
$$

where we denoted $e_{p}=p / p_{0}-\left(p / p_{0}\right)_{d}$. In order to study the stability of the system (45), (46) we propose the following Lyapunov function candidate

$$
V=\frac{J_{e}}{2} \widetilde{\omega}^{2}+\frac{1}{2} e_{p}^{2}
$$

Differentiating (47) along the solutions of (45), (46) we get

$$
\dot{V} \leqslant-\alpha_{1} \widetilde{\omega}^{2}+|\widetilde{\omega}|\left(-\frac{a_{2} k \gamma_{s}}{p_{0}}+|e|\right)+|\widetilde{\omega}|\left|e_{p}\right| a_{2}-\alpha_{2} e_{p}^{2}
$$

Now it is clear how to choose $\gamma_{s}$. Substituting the bound (26) in the derivative of the Lyapunov function we choose $\gamma_{s}$ as

$$
\gamma_{s}=\frac{p_{0}}{a_{2} k} \sqrt{e^{2}(0) e^{-\alpha_{0} t}+\frac{c_{1}^{2}}{\alpha_{0}^{2}}}
$$

Notice that, the power of the spark advance as a control is limited and $\gamma_{s}$ should be chosen sufficiently small so as not to force the spark advance into saturation. This can be achieved by choosing sufficiently large $\alpha_{0}$, or in other words, for any sufficiently small $\gamma_{s}$ there exists $\alpha_{0}$ satisfying (49). Notice also that, the estimation error (26) gets smaller via amplifying the design parameter $\alpha_{0}$. Substituting (49) in (48) we get

$$
\dot{V} \leqslant \widetilde{\omega}^{2}\left(-\alpha_{1}+\frac{a_{2}}{2}\right)+e_{p}^{2}\left(-\alpha_{2}+\frac{a_{2}}{2}\right)
$$

If the algorithm parameters are chosen in order to satisfy the following inequalities

$$
\begin{gathered}
\alpha_{1}>\frac{a_{2}}{2}+\kappa \frac{J_{e}}{2} \\
\alpha_{2}>\frac{a_{2}}{2}+\frac{\kappa}{2}
\end{gathered}
$$

where $\kappa>0$, then the following inequality holds

$$
\dot{V} \leqslant-\kappa V
$$

and our control aim (21) is reached.

\section{Simulation Results}

We simulated the system (18)-(20) with throttle control (43) and spark advance control (33), (34). We tested our algorithm under the following disturbances:

$$
T_{d}(t)= \begin{cases}0 & \text { if } t<2.5[\mathrm{sec}] \\ 20 & \text { otherwise }\end{cases}
$$

Simulation results are presented in Figs. 2 and 3.

Figure 2 demonstrates the performance of the disturbance estimation. Idle speed control performance together with the control variables, throttle and spark advance, are demonstrated in Fig. 3. It is worth remarking that the separation between $b$ and $u_{2}$ in our spark control loop is artificial, and despite the fact that it plays an important role in our control design procedure, only the sum $-b+u_{2}$ gives information about the position of spark advance from MBT (if $-b+u_{2}=0$ then the spark advance is at MBT). 


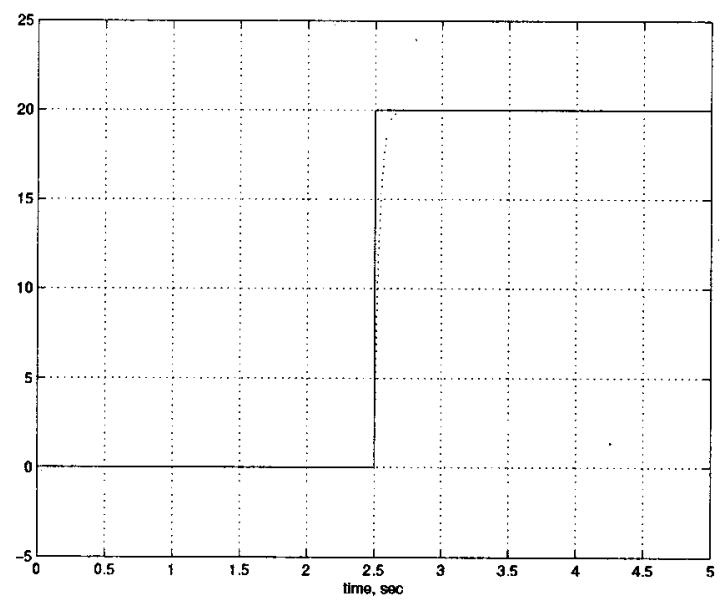

Fig. 2 Disturbance $(\mathrm{Nm})$ : solid line. Disturbance estimate $(N m)$ : dotted line.

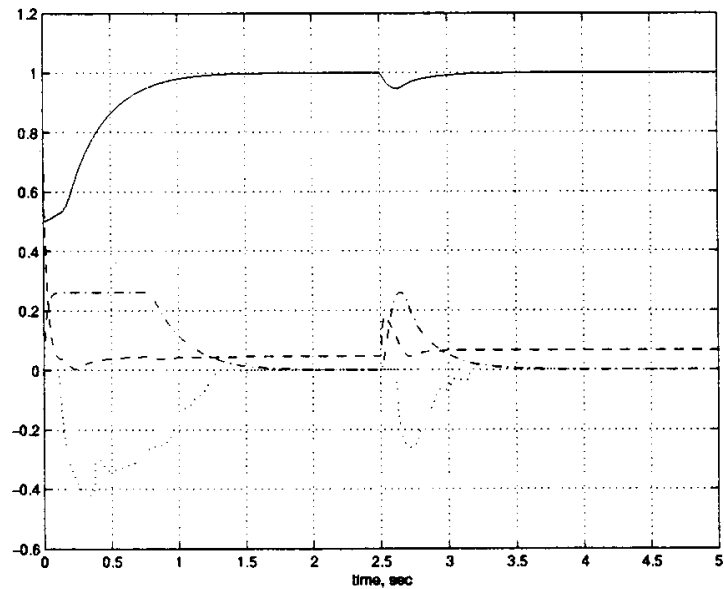

Fig. 3 Engine speed $\omega / 100(\mathrm{rad} / \mathrm{sec})$ : solid line. Throttle position: dashed line $-b+u_{2}(\mathrm{rad}):$ dotted line. Position of the axis $b(\mathrm{rad})$ : dash-dotted line.

\section{Conclusion}

In this paper we proposed a new solution for the ISC problem under unknown time varying disturbances. Our solution is based on explicit identification of the unknown disturbance and uses advantages and compensates disadvantages of throttle and spark advance as controls. The result allows the improvement of the performance of ISC systems.

\section{References}

[1] Hrovat, D., and Sun, J., 1997, "'Models and control methodologies for IC engine idle speed control design,' Control Eng. Practice, 5, No. 8, pp. 1093 1100

[2] Hrovat, D., Colvin, D., and Powell, B. K., 1998, “Comments on 'Applications of Some New Tools to Robust Stability Analysis of Spark Ignition Engine: A case Study, ," IEEE Trans. on Control System Technology, 6, No. 3, May.

[3] Green, J., and Hedrick, J. K., 1990, "Nonlinear Speed Control for Automotive Engines," Proc. of the 1990 ACC, San Diego, CA, May, pp. 2891-2897.

[4] Choi, S., and Hedrick, K., 1996, "Robust Throttle Control of Automotive Engines: Theory and Experiment," ASME J. Dyn. Syst., Meas., Control, 118 Mar., pp. 92-98.

[5] Eun, Y., Kim, J., Kim, K., and Cho, D., 1999, “'Discrete Time Variable Structure Controller with a Decoupled Disturbance Compensator and its Application to a CNC Servomechanism,' IEEE Trans. on Control System Technology, 7, No. 14, July.

[6] Kaynak, O., and Denker, A., 1993, "Discrete time sliding mode control in the presence of system uncertainty," Int. J. Control, 57, No. 5, pp. 1177-1189.

[7] Stotsky, A. A., 1995, "Performance Improvement in Indirect Adaptive Control Scheme in the Presence of Disturbances," Proc. 34 CDC, New Orleans, Louisiana, USA, Dec. 13-15.

[8] Cho, D., and Hedrick, K., 1989, “Automotive Powertrain Modeling for Control,’ ASME J. Dyn. Syst., Meas., Control, 111, Dec., pp. 568-576.

[9] Persson, U., 1995, “Engine Management System," Volvo Technical Report, 2102CHTK/CSE1.

[10] Kao, M., and Moskwa, J., 1995, “Turbo charged Diesel Engine Modeling for Nonlinear Engine Control and State Estimation,' ASME J. Dyn. Syst., Meas., Control, 117, Mar., pp. 20-30.

[11] Moskwa, J., and Hedrick, K., 1989, Sliding mode control of automotive engines, "Proceedings of the 1989 American Control Conference, American Autom. Control Council, Green Valley, AZ, Vol. 3, 2848, pp. p.1040-5, Vol. 2 .

[12] Drakunov, S., and Utkin, V., 1995, "Sliding Mode Observers. Tutorial,', Proc. 34-th CDC, New Orleans, pp. 3376-3378.

[13] Stotsky, A., Hedrick, K., and Yip, P., 1998, “The Use of Sliding Modes to Simplify Backstepping Control Method," Appl. Math. Comp. Sci., 8, No. 1, pp. $123-133$. 